\title{
A Novel Merchant Optimization Algorithm for SOlving Optimal REACTIVE POWER PROBLEM
}

\author{
Submitted: $6^{\text {th }}$ July 2019; accepted: $14^{\text {th }}$ March 2021
}

\section{K. Lenin}

DOI: 10.14313/JAMRIS/1-2021/7

\begin{abstract}
In this paper Merchant Optimization Algorithm (MOA) is proposed to solve the optimal reactive power problem. Projected algorithm is modeled based on the behavior of merchants who gain in the market through various mode and operations. Grouping of the traders will be done based on their specific properties, and by number of candidate solution will be computed to individual merchant. First Group named as "Ruler candidate solution" afterwards its variable values are dispersed to the one more candidate solution and it named as "Serf candidate solution" In standard IEEE 14, 30, 57 bus test systems Merchant Optimization Algorithm (MOA) have been evaluated. Results show the proposed algorithm reduced power loss effectively.
\end{abstract}

Keywords: Optimal reactive power, Transmission loss, Merchant Optimization Algorithm

\section{Introduction}

Reactive power problem plays a key role in secure and economic operations of power system. Optimal reactive power problem has been solved by variety of types of methods ([1-6]) . Nevertheless numerous scientific difficulties are found while solving problem due to an assortment of constraints. Evolutionary techniques ([7-16]) are applied to solve the reactive power problem, but the main problem is many algorithms get stuck in local optimal solution \& failed to balance the Exploration \& Exploitation during the search of global solution. In this paper Merchant Optimization Algorithm (MOA) has been proposed to solve the optimal reactive power problem. Projected algorithm is modeled based on the behavior of merchants who gain in the market through various mode and operations. Initial point of the projected algorithm all the merchants will possess same properties and in subsequent iterations properties will be updated. Then grouping of the traders will be done based on their specific properties, and by number of candidate solution will be computed to individual merchant. Subsequent to grouping of candidate solutions, then the most excellent candidate solution of each group named as "Ruler candidate solution" afterwards its variable values are dispersed to the one more candidate solution and it named as "Serf candidate solution". In standard IEEE 14, 30, 57 bus test systems [17] the proposed Merchant Optimization Algorithm (MOA) is evaluated. Simulation study shows the projected algorithm reduced power loss effectively.

\section{Problem Formulation}

True power loss reduction is main objective of the problem:

$$
\mathrm{F}=\mathrm{P}_{\mathrm{L}}=\sum_{(\mathrm{k} \in \mathrm{Nbr})} \mathrm{g}_{\mathrm{k}}\left(\mathrm{V}_{\mathrm{i}}^{2}+\mathrm{V}_{\mathrm{j}}^{2}-2 \mathrm{~V}_{\mathrm{i}} \mathrm{V}_{\mathrm{j}} \cos _{\mathrm{ij}}\right)
$$

Voltage deviation given as follows:

$$
\mathrm{F}=\mathrm{P}_{\mathrm{L}}+\omega_{\mathrm{v}} \times \text { Voltage Deviation }
$$

Voltage deviation given by:

$$
\text { Voltage Deviation }=\sum_{\mathrm{i}=1}^{\mathrm{Npq}}\left|\mathrm{V}_{\mathrm{i}}-1\right|
$$

Constraint (Equality)

$$
P_{G}=P_{D}+P_{L}
$$

Constraints (Inequality)

$$
\begin{gathered}
\mathrm{P}_{\text {gslack }}^{\min } \leq \mathrm{P}_{\text {gslack }} \leq \mathrm{P}_{\text {gslack }}^{\max } \\
\mathrm{Q}_{\mathrm{gi}}^{\min } \leq \mathrm{Q}_{\mathrm{gi}} \leq \mathrm{Q}_{\mathrm{gi}}^{\max }, \mathrm{i} \in \mathrm{N}_{\mathrm{g}} \\
\mathrm{V}_{\mathrm{i}}^{\min } \leq \mathrm{V}_{\mathrm{i}} \leq \mathrm{V}_{\mathrm{i}}^{\max }, \mathrm{i} \in \mathrm{N} \\
\mathrm{T}_{\mathrm{i}}^{\min } \leq \mathrm{T}_{\mathrm{i}} \leq \mathrm{T}_{\mathrm{i}}^{\max }, \mathrm{i} \in \mathrm{N}_{\mathrm{T}} \\
\mathrm{Q}_{\mathrm{c}}^{\min } \leq \mathrm{Q}_{\mathrm{c}} \leq \mathrm{Q}_{\mathrm{C}}^{\max }, \mathrm{i} \in \mathrm{N}_{\mathrm{C}}
\end{gathered}
$$

\section{Merchant Optimization Algorithm}

Merchant Optimization Algorithm (MOA) has been modeled based on the behavior of merchants who gain in the market through various mode and operations.

Initialization of population through candidate solution (CS) is done through various variables and given by,

$$
\text { variousvariables }=\left\{v v_{1}, v v_{2}, . ., v v_{n}, G\right\}
$$


Subsequent to the formation of the population the merit is computed through the objective function and with reference to the problem merit will be defined.

Initial point of the projected algorithm all the merchants will possess same properties and in subsequent iterations properties will be updated. Then grouping of the traders will be done based on their specific properties, then by following equation the number of candidate solution will be computed to individual merchant,

Totalnumber $_{i}=2+$ round $\left[\frac{Q_{i}}{\sum_{j=1}^{T} Q_{J}} \times(G-2 \times T)\right]$

Subsequent to grouping of candidate solutions, then the most excellent candidate solution of each group named as "Ruler candidate solution" afterwards its variable values are dispersed to the one more candidate solution and it named as "Serf candidate solution" by,

$\sum_{j=1}^{C k}\left(\sum_{i=1}^{R}(\right.$ candidate solution_Serf_j(random $\left.)(\mathrm{n})\right)=$

candidate solution_Ruler_k(random)(n)))

"Serf candidate solution" is improved by,

$\sum_{i=1}^{R}\left(\right.$ candidate solution $_{\operatorname{Serf}(R)}=$

candidate solution $_{\operatorname{Serf}(R)}+$

$+k \times$ random(candidate solution $\operatorname{serf}(R))$ )

To "Ruler candidate solution" most excellent candidate solution are imported which has been arbitrarily chosen,

$\sum_{j=1}^{T} \sum_{i=1}^{R}($ candidate solution_Ruler_j(random $(\mathrm{n}))=$ = candidate solution_Ruler_k(random(n))

$K=$

$=\{a \mid a \neq j$ and $a$ is an integer random value in $1 \leq a \leq n\}$

Merchant group's properties has been updated by,

$\operatorname{properties}_{i}=\left\{\sum_{j=1}^{B} O F(j) \mid\right.$ candidate $\left.\operatorname{solution}(\mathrm{j}, \mathrm{P})=\mathrm{P}_{\mathrm{i}}\right\}$

a. Initialization of parameters

b. Candidate population initiated

For $\mathrm{I}=1$ to $\mathrm{B}$

For $\mathrm{j}=1$ to $\mathrm{n}$

Candidate solution $(\mathrm{I}, \mathrm{j})=$ an arbitrary in defined level

For $\mathrm{I}=1$ to $\mathrm{T}$

Modernize the total number (I) by c. Objective function called

For $\mathrm{I}=1$ to $\mathrm{B}$

Candidate solution (I). Gain = Returned value form the objective function

d. Candidate solution grouping

$\mathrm{TMP}=\mathrm{NB}$

For $\mathrm{I}=1$ to $\mathrm{B}$

$k=\operatorname{random}(\operatorname{light}(T M P)$,

Candidate solution $(\mathrm{I}, \mathrm{n}+1)=\mathrm{k}$,

TMP $(\mathrm{k})$ - TMP $(\mathrm{k})-1$

if $\operatorname{TMP}(\mathrm{k})==0$ then $\operatorname{TMP}(:, \mathrm{k})=[\mathrm{]}$

e. Altering the candidate solution using

For $\mathrm{I}=1$ to $\mathrm{T}$

Ruler = most excellent candidate solution of the "I" group

For $\mathrm{J}=1$ to $\mathrm{B}$

Dispense "Ruler candidate solution" values to "Serf candidate solution" only if objective function value enhanced or else disregard the alteration

f. Altering the candidate solution using

For $\mathrm{I}=1$ to $\mathrm{B}$

Modernize the "Serf candidate solution" only if objective function value enhanced or else disregard the alteration

g. Altering the candidate solution using Modernize the "Ruler candidate solution" only if objective function value enhanced or else disregard the alteration

h. Modernization of the properties

Merchant properties are updated by

Stop if the end criterion is satisfied or else go to step " $d$ "

i. Output the best solution

\section{Simulation Results}

In standard IEEE 14 bus system validity of Merchant Optimization Algorithm (MOA) has been tested, Tab. 1 gives the constraints of control variables, Tab. 2 provides the limits of reactive power generators. Tab. 3 shows the comparison results.

Tab. 1. Control variables limits

\begin{tabular}{|c|c|c|c|}
\hline \multirow{2}{*}{ System } & Variables & $\begin{array}{c}\text { Minimum } \\
\text { (PU) }\end{array}$ & $\begin{array}{c}\text { Maximum } \\
\text { (PU) }\end{array}$ \\
\hline \multirow{2}{*}{$\begin{array}{c}\text { IEEE 14 } \\
\text { Bus }\end{array}$} & Generator Voltage & 0.95 & 1.1 \\
\cline { 2 - 4 } & Transformer Tap & 0.9 & 1.1 \\
\cline { 2 - 4 } & VAR Source & 0 & 0.20 \\
\hline
\end{tabular}

Tab. 2. Reactive power generators limits

\begin{tabular}{|c|c|c|c|}
\hline System & Variables & $\begin{array}{c}\text { Q Minimum } \\
\text { (PU) }\end{array}$ & $\begin{array}{c}\text { Q Maximum } \\
\text { (PU) }\end{array}$ \\
\hline \multirow{2}{*}{$\begin{array}{c}\text { IEEE 14 } \\
\text { Bus }\end{array}$} & 1 & 0 & 10 \\
\cline { 2 - 4 } & 2 & -40 & 50 \\
\cline { 2 - 4 } & 3 & 0 & 40 \\
\hline & 6 & -6 & 24 \\
\hline & 8 & -6 & 24 \\
\hline
\end{tabular}


Tab. 3. Simulation results of IEEE -14 system

\begin{tabular}{|l|c|c|c|c|c|c|}
\hline \multicolumn{1}{|c|}{ Control variables } & Base case & MPSO $[18]$ & $\begin{array}{c}\text { PSO } \\
{[18]}\end{array}$ & $\begin{array}{c}\text { EP } \\
{[18]}\end{array}$ & SARGA $[18]$ & MOA \\
\hline$V G-1$ & 1.060 & 1.100 & 1.100 & NR$^{*}$ & NR$^{*}$ & 1.024 \\
\hline$V G-2$ & 1.045 & 1.085 & 1.086 & 1.029 & 1.060 & 1.038 \\
\hline$V G-3$ & 1.010 & 1.055 & 1.056 & 1.016 & 1.036 & 1.037 \\
\hline$V G-6$ & 1.070 & 1.069 & 1.067 & 1.097 & 1.099 & 1.023 \\
\hline$V G-8$ & 1.090 & 1.074 & 1.060 & 1.053 & 1.078 & 1.035 \\
\hline Tap 8 & 0.978 & 1.018 & 1.019 & 1.04 & 0.95 & 0.940 \\
\hline Tap 9 & 0.969 & 0.975 & 0.988 & 0.94 & 0.95 & 0.942 \\
\hline Tap 10 & 0.932 & 1.024 & 1.008 & 1.03 & 0.96 & 0.946 \\
\hline$Q C-9$ & 0.19 & 14.64 & 0.185 & 0.18 & 0.06 & 0.131 \\
\hline$P G$ & 272.39 & 271.32 & 271.32 & $\mathrm{NR}^{*}$ & NR & 271.90 \\
\hline$Q R$ (Mvar) & 82.44 & 75.79 & 76.79 & $\mathrm{NR}^{*}$ & NR* & 75.91 \\
\hline Reduction in PLoss (\%) & 0 & 9.2 & 9.1 & 1.5 & 2.5 & 25.40 \\
\hline Total PLoss (Mw) & 13.550 & 12.293 & 12.315 & 13.346 & 13.216 & 10.108 \\
\hline
\end{tabular}

NR* - Not reported.

Then the proposed Merchant Optimization Algorithm (MOA) is evaluated in standard IEEE 30 Bus system. Tab. 4 gives the constraints of control variables, Tab. 5 shows the limits of reactive power generators and in Tab. 6 comparison of real power loss has been given.

Tab. 4. Constraints of control variables

\begin{tabular}{|c|c|c|c|}
\hline System & Variables & $\begin{array}{c}\text { Minimum } \\
\text { (PU) }\end{array}$ & $\begin{array}{c}\text { Maximum } \\
\text { (PU) }\end{array}$ \\
\hline \multirow{2}{*}{$\begin{array}{c}\text { IEEE 30 } \\
\text { Bus }\end{array}$} & Generator Voltage & 0.95 & 1.1 \\
\cline { 2 - 4 } & Transformer Tap & 0.9 & 1.1 \\
\cline { 2 - 4 } & VAR Source & 0 & 0.20 \\
\hline
\end{tabular}

Tab. 5. Constrains of reactive power generators

\begin{tabular}{|c|c|c|c|}
\hline System & Variables & $\begin{array}{c}\text { Q Minimum } \\
\text { (PU) }\end{array}$ & $\begin{array}{c}\text { Q Maximum } \\
\text { (PU) }\end{array}$ \\
\hline \multirow{2}{*}{$\begin{array}{c}\text { IEEE 30 } \\
\text { Bus }\end{array}$} & 1 & 0 & 10 \\
\cline { 2 - 4 } & 2 & -40 & 50 \\
\cline { 2 - 4 } & 5 & -40 & 40 \\
\hline & 8 & -10 & 40 \\
\hline & 11 & -6 & 24 \\
\hline & 13 & -6 & 24 \\
\hline
\end{tabular}

Tab. 6. Simulation results of IEEE -30 system

\begin{tabular}{|c|c|c|c|c|c|c|}
\hline Control variables & Base case & $\begin{array}{c}\text { MPSO } \\
{[18]}\end{array}$ & $\begin{array}{l}\text { PSO } \\
{[18]}\end{array}$ & $\begin{array}{c}\mathbf{E P} \\
{[18]}\end{array}$ & $\begin{array}{c}\text { SARGA } \\
{[18]}\end{array}$ & MOA \\
\hline$V G-1$ & 1.060 & 1.101 & 1.100 & $\mathrm{NR}^{*}$ & $\mathrm{NR}^{*}$ & 1.023 \\
\hline$V G-2$ & 1.045 & 1.086 & 1.072 & 1.097 & 1.094 & 1.030 \\
\hline$V G-5$ & 1.010 & 1.047 & 1.038 & 1.049 & 1.053 & 1.021 \\
\hline$V G-8$ & 1.010 & 1.057 & 1.048 & 1.033 & 1.059 & 1.042 \\
\hline$V G-12$ & 1.082 & 1.048 & 1.058 & 1.092 & 1.099 & 1.045 \\
\hline$V G-13$ & 1.071 & 1.068 & 1.080 & 1.091 & 1.099 & 1.037 \\
\hline Tap 11 & 0.978 & 0.983 & 0.987 & 1.01 & 0.99 & 0.941 \\
\hline Tap 12 & 0.969 & 1.023 & 1.015 & 1.03 & 1.03 & 0.943 \\
\hline Tap 15 & 0.932 & 1.020 & 1.020 & 1.07 & 0.98 & 0.930 \\
\hline Tap 36 & 0.968 & 0.988 & 1.012 & 0.99 & 0.96 & 0.941 \\
\hline$Q C 10$ & 0.19 & 0.077 & 0.077 & 0.19 & 0.19 & 0.090 \\
\hline QC 24 & 0.043 & 0.119 & 0.128 & 0.04 & 0.04 & 0.122 \\
\hline$P G(\mathrm{MW})$ & 300.9 & 299.54 & 299.54 & $\mathrm{NR}^{*}$ & $\mathrm{NR}^{*}$ & 297.73 \\
\hline$Q C$ (Mvar) & 133.9 & 130.83 & 130.94 & $\mathrm{NR}^{*}$ & $\mathrm{NR}^{*}$ & 131.44 \\
\hline Reduction in PLoss (\%) & 0 & 8.4 & 7.4 & 6.6 & 8.3 & 18.88 \\
\hline Total PLoss (Mw) & 17.55 & 16.07 & 16.25 & 16.38 & 16.09 & 14.241 \\
\hline
\end{tabular}


Then the Proposed Merchant Optimization Algorithm (MOA) has been tested, in IEEE 57 Bus system. Tab. 7 shows the constraints of control variables, Tab. 8 shows the limits of reactive power generators and comparison results are presented in Tab. 9.

Tab. 7. Constraints of control variables

\begin{tabular}{|c|c|c|c|}
\hline & Variables type & $\begin{array}{c}\text { Minimum } \\
\text { value (PU) }\end{array}$ & $\begin{array}{c}\text { Maximum } \\
\text { value (PU) }\end{array}$ \\
\hline \multirow{2}{*}{$\begin{array}{c}\text { IEEE 57 } \\
\text { Bus }\end{array}$} & Generator Voltage & 0.95 & 1.1 \\
\cline { 2 - 4 } & Transformer Tap & 0.9 & 1.1 \\
\cline { 2 - 4 } & VAR Source & 0 & 0.20 \\
\hline
\end{tabular}

Tab. 8. Constrains of reactive power generators

\begin{tabular}{|c|c|c|c|}
\hline \multirow{4}{*}{$\begin{array}{c}\text { IEEE 57 } \\
\text { Bus }\end{array}$} & Variables & $\begin{array}{c}\text { Q Minimum } \\
\text { (PU) }\end{array}$ & $\begin{array}{c}\text { Q Maximum } \\
\text { (PU) }\end{array}$ \\
\cline { 2 - 4 } & 1 & -140 & 200 \\
\cline { 2 - 4 } & 2 & -17 & 50 \\
\cline { 2 - 4 } & 3 & -10 & 60 \\
\cline { 2 - 4 } & 6 & -8 & 25 \\
\cline { 2 - 4 } & 8 & -140 & 200 \\
\cline { 2 - 4 } & 9 & -3 & 9 \\
\hline
\end{tabular}

Tab. 9. Simulation results of IEEE -57 system

\begin{tabular}{|c|c|c|c|c|c|c|}
\hline Control variables & Base case & $\begin{array}{c}\text { MPSO } \\
\text { [18] }\end{array}$ & $\begin{array}{l}\text { PSO } \\
\text { [18] }\end{array}$ & $\begin{array}{l}\text { CGA } \\
\text { [18] }\end{array}$ & $\begin{array}{l}\text { AGA } \\
{[18]}\end{array}$ & MOA \\
\hline$V G 1$ & 1.040 & 1.093 & 1.083 & 0.968 & 1.027 & 1.020 \\
\hline$V G 2$ & 1.010 & 1.086 & 1.071 & 1.049 & 1.011 & 1.022 \\
\hline$V G 3$ & 0.985 & 1.056 & 1.055 & 1.056 & 1.033 & 1.023 \\
\hline$V G 6$ & 0.980 & 1.038 & 1.036 & 0.987 & 1.001 & 1.012 \\
\hline$V G 8$ & 1.005 & 1.066 & 1.059 & 1.022 & 1.051 & 1.024 \\
\hline$V G 9$ & 0.980 & 1.054 & 1.048 & 0.991 & 1.051 & 1.021 \\
\hline$V G 12$ & 1.015 & 1.054 & 1.046 & 1.004 & 1.057 & 1.030 \\
\hline Tap 19 & 0.970 & 0.975 & 0.987 & 0.920 & 1.030 & 0.900 \\
\hline Tap 20 & 0.978 & 0.982 & 0.983 & 0.920 & 1.020 & 0.901 \\
\hline Tap 31 & 1.043 & 0.975 & 0.981 & 0.970 & 1.060 & 0.924 \\
\hline Tap 35 & 1.000 & 1.025 & 1.003 & $\mathrm{NR}^{*}$ & $\mathrm{NR}^{*}$ & 1.012 \\
\hline Tap 36 & 1.000 & 1.002 & 0.985 & $\mathrm{NR}^{*}$ & $\mathrm{NR}^{*}$ & 1.026 \\
\hline Tap 37 & 1.043 & 1.007 & 1.009 & 0.900 & 0.990 & 1.025 \\
\hline Tap 41 & 0.967 & 0.994 & 1.007 & 0.910 & 1.100 & 0.917 \\
\hline Tap 46 & 0.975 & 1.013 & 1.018 & 1.100 & 0.980 & 1.024 \\
\hline Tap 54 & 0.955 & 0.988 & 0.986 & 0.940 & 1.010 & 0.932 \\
\hline Tap 58 & 0.955 & 0.979 & 0.992 & 0.950 & 1.080 & 0.930 \\
\hline Tap 59 & 0.900 & 0.983 & 0.990 & 1.030 & 0.940 & 0.941 \\
\hline Tap 65 & 0.930 & 1.015 & 0.997 & 1.090 & 0.950 & 1.042 \\
\hline Tap 66 & 0.895 & 0.975 & 0.984 & 0.900 & 1.050 & 0.913 \\
\hline Tap 71 & 0.958 & 1.020 & 0.990 & 0.900 & 0.950 & 1.022 \\
\hline Tap 73 & 0.958 & 1.001 & 0.988 & 1.000 & 1.010 & 1.034 \\
\hline Tap 76 & 0.980 & 0.979 & 0.980 & 0.960 & 0.940 & 0.941 \\
\hline Tap 80 & 0.940 & 1.002 & 1.017 & 1.000 & 1.000 & 1.020 \\
\hline$Q C 18$ & 0.1 & 0.179 & 0.131 & 0.084 & 0.016 & 0.133 \\
\hline$Q C 25$ & 0.059 & 0.176 & 0.144 & 0.008 & 0.015 & 0.141 \\
\hline$Q C 53$ & 0.063 & 0.141 & 0.162 & 0.053 & 0.038 & 0.101 \\
\hline$P G(\mathrm{MW})$ & 1278.6 & 1274.4 & 1274.8 & 1276 & 1275 & 1272.10 \\
\hline$Q C$ (Mvar) & 321.08 & 272.27 & 276.58 & 309.1 & 304.4 & 272.23 \\
\hline Reduction in PLoss (\%) & 0 & 15.4 & 14.1 & 9.2 & 11.6 & 26.57 \\
\hline Total PLoss (Mw) & 27.8 & 23.51 & 23.86 & 25.24 & 24.56 & 20.412 \\
\hline
\end{tabular}

NR* - Not reported. 


\section{Conclusion}

In this paper Merchant Optimization Algorithm (MOA) solved the optimal reactive power problem effectively. Projected algorithm is modeled based on the behavior of merchants who gain in the market through various mode and operations. Initially all the merchants will possess the same properties and in subsequent iterations properties will be updated. Then grouping of the traders has been done based on their explicit properties. Proposed Merchant Optimization Algorithm (MOA) has been tested in standard IEEE 14, 30, 57 bus test systems and simulation results show the projected algorithm reduced the real power loss. Percentage of real power loss reduction has been improved.

\section{AUTHOR}

Kanagasabai Lenin - Department of Electrical and Electronics Engineering, Prasad V. Potluri Siddhartha Institute of Technology, Vijayawada, India, e-mail: gklenin@gmail.com.

\section{REFERENCES}

[1] K. Y. Lee, Y. M. Park and J. L. Ortiz, "Fuel-cost minimisation for both real- and reactive-power dispatches", IEE Proceedings C (Generation, Transmission and Distribution), vol. 131, no. 3, 1984, 85-93, 10.1049/ip-c.1984.0012.

[2] N. I. Deeb and S. M. Shahidehpour, "An Efficient Technique for Reactive Power Dispatch Using a Revised Linear Programming Approach", Electric Power Systems Research, vol. 15, no. 2, 1988, 121-134, 10.1016/0378-7796(88)90016-8.

[3] M. Bjelogrlic, M. S. Calovic, P. Ristanovic and B. S. Babic, "Application of Newton's optimal power flow in voltage/reactive power control", IEEE Transactions on Power Systems, vol. 5, no. 4, 1990, 1447-1454, 10.1109/59.99399.

[4] S. Granville, "Optimal reactive dispatch through interior point methods", IEEE Transactions on Power Systems, vol. 9, no. 1, 1994, 136-146, 10.1109/59.317548.

[5] N. Grudinin, "Reactive power optimization using successive quadratic programming method", IEEE Transactions on Power Systems, vol. 13, no. 4, 1998, 1219-1225, 10.1109/59.736232.

[6] R. Ng Shin Mei, M. H. Sulaiman, Z. Mustaffa and H. Daniyal, "Optimal reactive power dispatch solution by loss minimization using moth-flame optimization technique", Applied Soft Computing, vol. 59, 2017, 210-222, 10.1016/j. asoc.2017.05.057.
[7] G. Chen, L. Liu, Z. Zhang and S. Huang, "Optimal reactive power dispatch by improved GSA-based algorithm with the novel strategies to handle constraints", Applied Soft Computing, vol. 50, 2017, 58-70, 10.1016/j.asoc.2016.11.008.

[8] E. Naderi, H. Narimani, M. Fathi and M. R. Narimani, "A novel fuzzy adaptive configuration of particle swarm optimization to solve large-scale optimal reactive power dispatch", Applied Soft Computing, vol. 53, 2017, 441-456, 10.1016/j. asoc.2017.01.012.

[9] A. A. Heidari, R. Ali Abbaspour and A. Rezaee Jordehi, "Gaussian bare-bones water cycle algorithm for optimal reactive power dispatch in electrical power systems", Applied Soft Computing, vol. 57, 2017, 657-671, 10.1016/j. asoc.2017.04.048.

[10] M. Mahaletchumi, A. Nor Rul Hasma, S. M. H., M. Mahfuzah and S. Rosdiyana, "Benchmark studies on Optimal Reactive Power Dispatch (ORPD) Based Multi-Objective Evolutionary Programming (MOEP) using Mutation Based on Adaptive Mutation Operator (AMO) and Polynomial Mutation Operator (PMO)", Journal of Electrical Systems, vol. 12, no. 1, 2016, 121-132.

[11] R. Ng Shin Mei, M. H. Sulaiman and Z. Mustaffa, "Ant lion optimizer for optimal reactive power dispatch solution", Journal of Electrical Systems - Special Issue AMPE2015, vol. 3, 2015, 68-74.

[12] P. Anbarasan and T. Jayabarathi, "Optimal reactive power dispatch problem solved by symbiotic organism search algorithm". In: 2017 Innovations in Power and Advanced Computing Technologies (i-PACT), 2017, 1-8, 10.1109/ IPACT.2017.8244970.

[13] A. Gagliano and F. Nocera, "Analysis of the performances of electric energy storage in residential applications", International Journal of Heat and Technology, vol. 35, no. Special Issue 1, 2017, S41-S48, 10.18280/ijht.35Sp0106.

[14] M. Caldera, P. Ungaro, G. Cammarata and G. Puglisi, "Survey-based analysis of the electrical energy demand in Italian households", Mathematical Modelling of Engineering Problems, vol. 5, no. 3, 2018, 217-224, 10.18280/mmep.050313.

[15] M. Basu, "Quasi-oppositional differential evolution for optimal reactive power dispatch", International Journal of Electrical Power \& Energy Systems, vol. 78, 2016, 29-40, 10.1016/j. ijepes.2015.11.067.

[16] G.-G. Wang, "Moth search algorithm: a bio-inspired metaheuristic algorithm for global optimization problems", Memetic Computing, vol. 10, 
no. 2 , 2018, 151-164, 10.1007/s12293-0160212-3.

[17] "Power Systems Test Case Archive". University of Washington, Electrical \& Computer Engineering, https://labs.ece.uw.edu/pstca/. Accessed on: 2021-06-28.

[18] A. N. Hussain, A. A. Abdullah and O. M. Neda, "Modified Particle Swarm Optimization for Solution of Reactive Power Dispatch", Research Journal of Applied Sciences, Engineering and Technology, vol. 15, no. 8, 2018, 316-327, 10.19026/ rjaset.15.5917. 